\title{
Verità e leggende sulla fistola arterovenosa
}

\author{
Carlo Basile, Carlo Lomonte
}

U.O.C. di Nefrologia e Dialisi, Ospedale Generale Miulli, Acquaviva delle Fonti (BA)

\begin{abstract}
Truths AND LEGENDS ON ARTERIOVENOUS FISTULA
Abstract. Large studies show a graded mortality risk from both cardiovascular (CV) and infectious diseases depending on access type, with the highest risk associated with catheters, followed by grafts, and then arteriovenous fistulas (AVF). The presence of an AVF has an adverse effect on cardiac function, but its exact contribution to CV morbidity is not clear. It has long been known that a vascular access (VA) with an inappropriately high-flow rate may be the cause of high-output heart failure. Even more paradoxically, there may be cardio-pulmonary benefits conferred by AVF. Thus, while emphasizing the real benefits of creating a native AVF, we would also like to stress the danger of attaining excessive blood flow rates. The key word in the case of VA choice is "eligibility". A "patient first, not fistula first, but avoid a catheter if at all possible" approach might be the best.
\end{abstract}

Key words: Arteriovenous fistula, Cardiovascular disease, Cardiovascular mortality, Congestive heart failure, Hemodialysis, High-output heart failure

\section{Conflict of interest: None.}

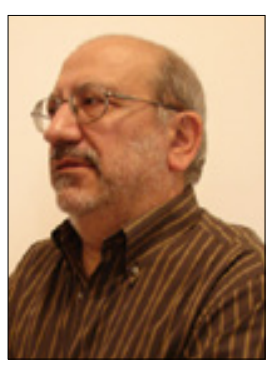

Carlo Basile

\section{Introduzione}

Il trattamento emodialitico (HD) a lungo termine richiede un accesso vascolare (VA) durevole al sistema circolatorio, con una bassa percentuale di complicanze e con un flusso di sangue (Qa) tale da garantire il target ottimale di dose dialitica (1). Se manca ancora l'evidenza degli studi randomizzati controllati, vi è, tuttavia, un ampio consenso sul fatto che il tipo di VA non solo contribuisce alla morbidità dei pazienti in $\mathrm{HD}$, ma può anche contribuire in modo indipendente alla loro mortalità (2-5). La fistola arterovenosa (FAV) nativa è considerata il migliore VA per la sua maggiore sopravvivenza e per le percentuali di complicanze inferiori rispetto ad altre forme di VA, come le protesi sintetiche arterovenose (AVG) e i cateteri venosi centrali (CVC) (25). Studi di grandi dimensioni dimostrano rischi di mortalità differenti, sia per cause cardiovascolari $(\mathrm{CV})$ che per malattie infettive, a seconda del tipo di VA, con il rischio più alto associato al CVC, quindi all'AVG e, infine, alla FAV (2-5). Per esempio, lo US Renal Data System Morbidity and Mortality Study Wave 2 condotto su 5507 pazienti prevalenti in HD aveva già dimostrato che $\mathrm{i}$ decessi per cause $\mathrm{CV}$ erano più elevati nei pazienti portatori di CVC rispetto a quelli portatori di FAV, sia diabetici che non diabetici (3). Una più recente analisi dello US Renal Data System Clinical Performance Measures, che includeva 4854 pazienti incidenti, ha dimostrato che l'utilizzo della FAV era fortemente associato a una più bassa mortalità da tutte la cause e da cause CV. Questo vantaggio era persistente anche dopo 4 anni ed era indipendente dall'effetto di altri fattori di rischio (2). Molte sono le possibili spiegazioni circa l'associazione tra l'utilizzo della FAV e la diminuzione del rischio di morte per eventi CV. Queste possono includere una maggiore dose somministrata di dialisi, un più elevato flusso di sangue $(\mathrm{Qa})$ della $\mathrm{FAV}$, un ridotto rischio di infezione e livelli più bassi di mediatori infiammatori nei pazienti che usano la FAV.

Infine, una recentissima review sistematica ha evidenziato che i pazienti portatori di CVC avevano un rischio più elevato di mortalità da tutte le cause (1.53), di infezioni fatali (2.12) e di eventi cardiovascolari (1.38) rispetto ai pazienti portatori di FAV. Similmente, quando confrontati con i pazienti portatori di $\mathrm{AVG}$, i pazienti portatori di $\mathrm{CVC}$ avevano un rischio più elevato di mortalità da tutte le cause (1.38), di infezioni fatali (1.49) e di eventi cardiovascolari (1.26). Rispetto ai pazienti portatori di FAV, i pazienti portatori di AVG avevano un rischio più elevato di mortalità da tutte le cause (1.18) e di infezioni fatali (1.36), ma non di eventi cardiovascolari (1.07) (6). Ancora più paradossalmente, ci possono essere effetti cardioprotettivi legati alla creazione di una FAV: un recente studio retrospettivo su 820 pazienti uremici cronici incidenti in HD trattati in tre città canadesi non evidenzia un aumento del rischio di morte per valori più elevati di Qa (7). Un altro studio ha dimostrato che i pazienti con Qa>1000 $\mathrm{mL} / \mathrm{min}$ hanno avuto una minore incidenza di ipertrofia ventricolare sinistra (LVH) e che livelli maggiori di Qa sembrano essere associati 
a un livello inferiore di stordimento (stunning) cardiaco indotto dall'HD (8). Infine, un altro effetto cardio-protettivo connesso alla creazione di una FAV è la riduzione della rigidità (stiffness) arteriosa $(9,10)$. È stato dimostrato che la rigidità aortica è un predittore indipendente di mortalità per tutte le cause e per cause CV nei pazienti in HD (11).

\section{Ipertensione polmonare e FAV}

L'ipertensione polmonare (PHT) è un aumento della pressione nell'arteria polmonare, nella vena polmonare o nei capillari polmonari, globalmente identificati come vascolarizzazione polmonare. Questa è un'alterazione emodinamica complessa che può derivare da cause disparate (polmonari, cardiache, sistemiche). La pressione polmonare è misurata inserendo un catetere multilume (catetere di Swan-Ganz) munito di un pallone all'estremità in una vena periferica, quindi avanzando il catetere nell'atrio destro, nel ventricolo destro, nell'arteria polmonare e, quindi, in un ramo dell'arteria polmonare. Gonfiando il pallone, esso occlude il ramo dell'arteria polmonare, rendendo, così, possibile la misurazione della cosiddetta pulmonary capillary wedge pressure (PCWP), che, di fatto, dà una misura indiretta della pressione nell'atrio sinistro. La PCWP aiuta a quantificare la gravità dello scompenso del ventricolo sinistro. Il sintomo principale della PHT è la dispnea, esacerbata ulteriormente dall'esercizio. La PHT può essere una grave malattia con una tolleranza all'esercizio marcatamente ridotta e con scompenso cardiaco (HF). La PHT è considerata essere un predittore indipendente di mortalità quasi uguale a quello associato alle cardiopatie severe nei pazienti in HD $(12,13)$. Sulla base di studi ecocardiografici, la prevalenza di PHT nei pazienti in HD è stimata essere intorno al 17$56 \%$ (14). A oggi, è stato pubblicato un solo studio sistematico che applica metodiche emodinamiche invasive (cateterismo cardiaco destro) nei pazienti affetti da malattia renale cronica (CKD), agli stadi 4 e 5, sia in pre-dialisi che in dialisi (14). Questo studio prospettico, denominato PEPPER ("prevalence of precapillary pulmonary arterial hypertension in patients with end-stage renal disease"), ha arruolato 31 pazienti agli stadi 4 e 5 di CKD, non ancora in dialisi, e 31 pazienti in dialisi, inviati consecutivamente a un Dipartimento accademico in Germania per dispnea inesplicata durante un'attività fisica ordinaria o per grave dispnea a riposo (14). Tra i criteri di esclusione dallo studio, va ricordato quello di una frazione di eiezione ventricolare sinistra $<50 \%$. Il primo dato importante dello studio è che 22/31 (71\%) pazienti agli stadi 4 e 5 di CKD non in dialisi avevano un'ipertensione polmonare postcapillare (Tab. I) (14). Quindi, contrariamente all'opinione corrente che la PHT nei pazienti in HD dipende principalmente da un'elevata gittata cardiaca (CO) secondaria alla presenza di una FAV, questo studio suggerisce che la PHT pre-esiste alla creazione della medesima in una cospicua percentuale di pazienti uremici cronici. L'ipertensione polmonare postcapillare fu evidenziata nel 65\% (20/31) dei pazienti in HD. Il secondo dato importante dello studio è che il $13 \%$ dei pazienti in dialisi (4/31) affetti da dispnea moderata-grave aveva un'ipertensione arteriosa polmonare idiopatica (ipertensione polmonare precapillare) (Tab. I) (14). Questo dato è stupefacente se si confronta con il dato corrispondente nella popolazione generale, che è di solo $15-50$ per milione nella popolazione adulta (15). Nello stesso studio nessun caso di ipertensione polmonare precapillare fu riscontrato nella serie parallela di 31 pazienti agli stadi 4 e 5 di CKD non in dialisi (14). Ne consegue che alterazioni ormonali e metaboliche associate allo stato di dialisi potrebbero contribuire alla vasocostrizione polmonare precapillare; tra queste si annoverano una funzione endoteliale ridotta, una diminuita disponibilità di ossido nitrico e aumentati livelli di endotelina (14). Infine, va sottolineato che, dato che lo studio PEPPER escludeva i pazienti con frazione di eiezione ventricola sinistra $<50 \%$, l'ipertensione polmonare postcapillare presente nel $65 \%$ dei pazienti in dialisi era attribuibile a una disfunzione diastolica del ventricolo sinistro (14). Lo scompenso cardiaco diastolico, chiamato anche HF con frazione di eiezione preservata, si riferisce a una sindrome clinica in cui i pazienti hanno segni e sintomi di HF, funzione sistolica del ventricolo sinistro normale o quasi normale ed evidenza di disfunzione diastolica (abnorme riempimento del ventricolo sinistro con elevate pressioni di riempimento) (16).

\section{La FAV ad alta portata}

Attualmente, non esiste una definizione univoca di FAV ad alta portata. Pandeya e Lindsey suggerirono di utilizzare il rapporto $\mathrm{Qa} / \mathrm{CO}$ : questo è il cosiddetto ricircolo cardio-polmonare (CPR). In uno studio a lungo termine essi trovarono che la media del Qa era pari a $1.6 \mathrm{~L} / \mathrm{min}$ e che la media della $\mathrm{CO}$ era pari a 7.2 $\mathrm{L} / \mathrm{min}$, con la conseguente media del CPR del $22 \%$ (17). Le Linee Guida della Vascular Access Society definiscono FAV ad alta portata quella il cui Qa è compreso tra 1.0 e $1.5 \mathrm{~L} / \mathrm{min}$ e con un CPR $>20 \%$ (18). Basile et al. (19) e van der Mark et al. (20) hanno dimostrato che un modello di regressione polinomiale di terzo ordine è quello che esprime meglio il rapporto tra $\mathrm{Qa}$ e CO. Inoltre, Basile et al. hanno dimostrato, in uno studio prospettico su 96 pazienti in $\mathrm{HD}$, che la $\mathrm{CO}$ non variava in modo significativo per valori di Qa compresi tra 0.95 e $2.2 \mathrm{~L} / \mathrm{min}$. In altre parole, l'aumento del Qa non era accompagnato da un aumento parallelo della CO (19). Le cause di questo fenomeno non sono note, ma si può ipotizzare una sorta di riserva funzionale del miocardio e, quindi, di adattamento del miocardio, capace di sostenere aumenti di Qa nel lungo termine senza la precipitazione di HF. Non sono disponibili in letteratura studi prospettici che studino le

TABELLA I - DISTINZIONE TRA IPERTENSIONE ARTERIOSA POLMONARE, IPERTENSIONE POLMONARE PRECAPILLARE E IPERTENSIONE POLMONARE POSTCAPILLARE

\begin{tabular}{llc}
\hline & $\begin{array}{c}\text { Pressione media } \\
\text { arteria } \\
\text { polmonare }\end{array}$ & PCWP \\
\hline $\begin{array}{l}\text { Assenza di ipertensione arteriosa } \\
\text { polmonare } \\
\text { Ipertensione arteriosa polmonare }\end{array}$ & $<25 \mathrm{mmHg}$ & \\
Ipertensione polmonare precapillare & $\geq 25 \mathrm{mmHg}$ & \\
Ipertensione polmonare postcapillare & $\geq 25 \mathrm{mmHg} \quad<15 \mathrm{mmHg}$ \\
\hline
\end{tabular}


variazioni del volume telediastolico ventricolare sinistro o della massa ventricolare sinistra in maniera prospettica e per periodi di tempo prolungati nei pazienti prima dell'inizio della dialisi o in dialisi. Purtroppo, l'unico modo per determinare se la creazione di una FAV determini LVH nei pazienti in HD è uno studio prospettico randomizzato di confronto tra CVC e FAV, che esamini le variazioni seriali delle dimensioni e delle performance del ventricolo sinistro. A causa della morbidità e della mortalità associate ai $\mathrm{CVC}$, è assolutamente improbabile che un simile studio possa essere progettato per motivi etici (21).

\section{FAV ad alta portata e HF}

La principale causa di morte nei pazienti in HD è un evento CV. Più del $50 \%$ dei soggetti trattati con HD periodica muore per malattie CV (22). La presenza della FAV ha un effetto negativo sulla funzione cardiaca, ma il suo esatto contributo alla morbidità $\mathrm{CV}$ non è chiaro.

L'HF è spesso associato a una riduzione della filtrazione glomerulare (GFR). La prevalenza di un'insufficienza renale moderata-grave (definita come una GFR $<60 \mathrm{~mL} / \mathrm{min}$ per 1.73 $\mathrm{m}^{2}$ di superficie corporea) è pari a circa il $30-60 \%$ in pazienti affetti da HF $(23,24)$. Le seguenti osservazioni sono esemplari: 1) in 80000 pazienti sia ospedalizzati che non ospedalizzati e affetti da HF, un'insufficienza renale da moderata a grave (definita come una GFR stimata pari a $<53 \mathrm{~mL} / \mathrm{min}$ e una creatininemia $>1.5 \mathrm{mg} / \mathrm{dL}$ ) è stata descritta nel $29 \%$ dei pazienti (23);2) nel database dell'Acute Decompensated Heart Failure National Registry, il 30\% circa di oltre 100000 pazienti ospedalizzati per HF ha avuto una diagnosi di CKD (definita come una creatininemia $>2.0 \mathrm{mg} / \mathrm{dL}$ ) (24). Lo scompenso cardiaco congestizio (CHF) è presente in più di un terzo dei pazienti che iniziano l'HD (25), con un'incidenza di 71/1000 anni-persona. Questa cifra è sostanzialmente maggiore dell'incidenza di sindromi coronariche acute allo stadio terminale della malattia renale cronica (ESRD) (29/1000 annipersona nello US Renal Data System Morbidity and Mortality Study Wave 2) (26). Il CHF nei pazienti in HD differisce dal CHF presente nei soggetti con funzionalità renale integra per diversi fattori: infatti, il sovraccarico di volume interdialitico e il Qa della FAV sono specifici solo dell'ESRD. Pertanto, l'HD contribuisce per sé allo sviluppo di CHF.

È noto da tempo che un VA con un Qa inappropriatamente elevato può essere la causa di HF ad alta gittata (27-33). Dopo la creazione di una FAV, il sangue viene deviato dal versante arterioso a pressione elevata al versante venoso a bassa pressione. Questa diversione di sangue verso la circolazione destra riduce il flusso arterioso sistemico. La risposta controregolatoria è un aumento della $\mathrm{CO}$ mediato dal sistema nervoso simpatico e dalle catecolamine circolanti. Inizialmente, il cuore aumenta la $\mathrm{CO}$ tramite un aumento della frequenza cardiaca e dello stroke volume. Con il tempo, l'eccessiva stimolazione cardiaca porta a $\mathrm{LVH}$, riduzione della frazione di eiezione ventricolare sinistra ed eventuale HF (31). Poca letteratura è disponibile per quanto riguarda l'HF ad alta gittata nei pazienti in HD, oltre ad alcuni case report (27-33). L'incidenza di questa complicanza non è nota: uno studio ha trovato un'incidenza pari al 3.7\% (17/460) di VA ad alto flusso richiedenti una correzione chirurgica (34). Quale (i) causa (e) è (sono) in grado di trasformare una LVH da sovraccarico di volume in HF non è noto. Caratteristiche specifiche dei pazienti o delle FAV (in particolare le dimensioni e la sede dell'anastomosi) o di entrambi possono predisporre allo sviluppo di HF (35). Alcuni Autori suggeriscono che uno scompenso cardiaco dovuto alla FAV possa svilupparsi solo nei pazienti con sottostante cardiopatia (36). Al momento, l'evidenza che lega la FAV allo sviluppo di HF è indiretta, ma è coerente con ciò che è noto per l'HF ad alta gittata che si verifica in altre condizioni patologiche, come le FAV traumatiche. I fattori di rischio per lo sviluppo di FAV ad alto Qa sono le dimensioni dell'anastomosi, il posizionamento della FAV al braccio e precedenti interventi chirurgici per la creazione del VA $(19,37)$. Il recente studio di Basile et al. ha dimostrato che le FAV posizionate nel braccio sono associate a un rischio aumentato di HF ad alta gittata (19). Anche se si deve riconoscere che le FAV dell'avambraccio di solito sono posizionate in un tipo di paziente con un fenotipo diverso rispetto ai pazienti che hanno una FAV nel braccio (tra i primi, di solito, ci sono meno diabetici e più giovani con meno malattie vascolari e disfunzioni cardiache), rimane il fatto che una simile associazione sembra favorire un ruolo causale delle FAV posizionate nel braccio nella patogenesi dell'HF ad alta gittata. Anche se è probabile che solo una piccola percentuale di pazienti in HD possa avere un CHF conclamato (uno studio ha sottolineato che solo il $2.6 \%$ dei pazienti con FAV al braccio è stato sottoposto a banding o a legatura della FAV per sindrome da furto o sindromi ad alta gittata) (38), il messaggio derivante da questo studio (19) è chiaro: le FAV agli arti superiori devono essere collocate il più distalmente possibile, come sottolineato anche dalle recenti Linee Guida EBPG (39).

\section{Verità e leggende sulla FAV}

Molto recentemente, Amerling et al. hanno scritto una review il cui principale messaggio era: "La FAV è un'anomalia non fisiologica e deve essere considerata un male minore... Indubbiamente, contribuisce all'eccessiva mortalità $\mathrm{CV}$ nei pazienti in HD e accorcia la durata della vita" (40). Abbiamo confutato le conclusioni di Amerling et al. (41, 42), la più importante delle quali era: "Anche pazienti giovani e relativamente sani svilupperanno complicanze $\mathrm{CV}$ e CHF, a causa della prolungata presenza di una FAV" (40). Questa affermazione non è supportata da alcuno studio; in realtà, è stato dimostrato che: a) anche una disfunzione renale lieve deve essere considerata una condizione medica predisponente a un aumento del rischio CV (43); b) l'insufficienza renale cronica è associata in maniera indipendente a un aumento della mortalità CV correlata alla malattia (44); c) i pazienti che iniziano l'HD sono già altamente preselezionati, perché le probabilità di morte sono molto più alte di quelle di arrivare al trattamento dialitico in tutte le fasi della CKD (45); d) ispessimento della parete arteriosa e rigidità aortica sono ben evidenti molto tempo prima dell'inizio dell'HD (46); e) solo il 16\% dei pazienti incidenti in HD aveva un ecocardiogramma normale (47); f) il tasso standardizzato di mortalità $\mathrm{CV}$ nei pazienti incidenti in $\mathrm{HD}$ era 38.1 volte per 1000 persone-anno più alto rispetto alla po- 
polazione generale (48). La FAV è un male minore (40) o una benedizione di Dio $(41,42)$ ? La risposta corretta è, ovviamente, la seconda. In realtà, la review di Amerling et al. (40) nega i benefici salvavita della FAV per milioni di esseri umani. Tuttavia, pur sottolineando i reali benefici della creazione di una FAV, vogliamo, in questa sede, sottolineare anche il pericolo legato a Qa elevati.

\section{Conclusioni}

Studi di grandi dimensioni dimostrano un rischio di mortalità progressivamente crescente a seconda del tipo di VA, con il rischio più alto associato al $\mathrm{CVC}$, seguito dai rischi legati all'AVG e, quindi, alla FAV (2-6). La presenza di una FAV ha un effetto negativo sulla funzione cardiaca, ma il suo esatto contributo alla morbidità $\mathrm{CV}$ non è chiaro. È noto da tempo che un VA con un Qa inappropriatamente elevato può essere la causa di HF ad alta gittata (27-33). Ancora più paradossalmente, ci possono essere benefici cardio-polmonari derivanti dalla presenza di una FAV (8-10). Tuttavia, pur sottolineando i reali benefici della creazione di una FAV, vogliamo anche sottolineare il pericolo legato a Qa elevati.

La parola chiave nella scelta di un VA è "eleggibilità" (49). Un approccio del tipo "al primo posto viene il paziente e non la FAV, ma è meglio evitare un CVC, se possibile" potrebbe essere la scelta migliore (1).

\section{Riassunto}

Studi di grandi dimensioni dimostrano un rischio di mortalità progressivamente crescente a seconda del tipo di accesso vascolare (VA), con il rischio più alto associato al catetere venoso centrale (CVC), seguito dai rischi associati alla protesi e, quindi, alla fistola arterovenosa (FAV). La presenza di una FAV ha un effetto negativo sulla funzione cardiaca, ma il suo esatto contributo alla morbidità cardiovascolare non è chiaro. È noto da tempo che un VA con un flusso inappropriatamente elevato può essere la causa di uno scompenso cardiaco ad alta gittata. Ancora più paradossalmente, ci possono essere benefici cardio-polmonari derivanti dalla presenza di una FAV. Tuttavia, pur sottolineando i reali benefici della creazione di una FAV, vogliamo anche sottolineare il pericolo legato a flussi elevati. La parola chiave nella scelta di un VA è "eleggibilità". Un approccio del tipo "al primo posto viene il paziente e non la FAV, ma è meglio evitare un CVC, se possibile" potrebbe essere la scelta migliore.

Parole Chiave: Emodialisi, Fistola arterovenosa, Malattia cardiovascolare, Mortalità cardiovascolare, Scompenso cardiaco ad alta gittata, Scompenso cardiaco congestizio

Dichiarazione di conflitto di interesse: Gli Autori dichiarano di non avere conflitto di interessi.

\section{Glossario}

$\mathrm{AVG}=$ protesi sintetica arterovenosa

$\mathrm{CHF}=$ scompenso cardiaco congestizio

$\mathrm{CKD}=$ malattia renale cronica

$\mathrm{CO}=$ gittata cardiaca

$\mathrm{CPR}=$ ricircolo cardio-polmonare

$\mathrm{CV}=$ cardiovascolare

$\mathrm{CVC}=$ catetere venoso centrale

$\mathrm{ESRD}=$ uremia cronica terminale

$\mathrm{FAV}=$ fistola arterovenosa

$\mathrm{GFR}=$ filtrato glomerulare

$\mathrm{HD}=$ emodialis

$\mathrm{HF}=$ scompenso cardiaco

$\mathrm{LVH}=$ ipertrofia ventricolare sinistra

PCWP = pressione capillare polmonare dopo incuneamento

PHT $=$ ipertensione polmonare

$\mathrm{Qa}=$ flusso di sangue nell'accesso vascolare

$\mathrm{VA}=$ accesso vascolare

Indirizzo degli Autori:

Dr. Carlo Basile

Via C. Battisti 192

74100 Taranto

basile.miulli@libero.it

\section{Bibliografia}

1. Besarab A. Resolved: fistulas are preferred to grafts as initial vascular access for dialysis. J Am Soc Nephrol 2008; 19: 1629-33.

2. Wasse H, Speckman RA, McClellan WM. Arteriovenous fistula use is associated with lower cardiovascular mortality compared with catheter use among ESRD patients. Semin Dial 2008; 21: 483-9.

3. Dinghra RK, Young EW, Hulbert-Shearon TE, et al. Type of vascular access and mortality in U.S. hemodialysis patients.
Kidney Int 2001; 60: 1443-51.

4. Astor BC, Eustace JA, Powe NR, et al. Type of vascular access and survival among incident hemodialysis patients: the choices for healthy outcomes in caring for ESRD (CHOICE) Study. J Am Soc Nephrol 2005; 16: 1449-55.

5. Ocak G, Halbesma N, le Cessie S, et al. Haemodialysis catheters increase mortality as compared to arteriovenous accesses especially in elderly patients. Nephrol Dial Transplant 2011; 26: 2611-7.

6. Ravani P, Palmer SC, Oliver MJ, et al. Associations between hemodialysis access type and clinical outcomes: a syste- 
matic review. J Am Soc Nephrol 2013; 24: 465-73.

7. Al-Ghonaim M, Manns BJ, Hirsch DJ, et al. Relation between access blood flow and mortality in chronic hemodialysis patients. Clin J Am Soc Nephrol 2008; 3: 387-91.

8. Korsheed S, Burton JO, McIntyre CW. Higher arteriovenous fistulae blood flows are associated with a lower level of dialysis-induced cardiac injury. Hemodial Int 2009; 13 : 505-511.

9. Utescu MS, LeBoeuf A, Chbinou N, et al. The impact of arteriovenous fistulas on aortic stiffness in patients with chronic kidney disease. Nephrol Dial Transplant 2009; 24: 3441-6.

10. Korsheed S, Eldehni MT, John SG. Effects of arteriovenous fistula formation on arterial stiffness and cardiovascular performance and function. Nephrol Dial Transplant 2011; 26: 3296-302.

11. Blacher J, Guerin AP, Pannier B, et al. Impact of aortic stiffness on survival in end-stage renal disease. Circulation 1999; 99: 2434-9.

12. Yigla M, Fruchter O, Aharson D, et al. Pulmonary hypertension is an independent predictor of mortality in hemodialysis patients. Kidney Int 2009; 75: 969-75.

13. Agarwal R. Prevalence, determinants and prognosis of pulmonary hypertension among hemodialysis patients. Nephrol Dial Transplant 2012; 27: 3908-14.

14. Pabst S, Hammerstingl C, Hundt F, et al. Pulmonary hypertension in patients with chronic kidney disease on dialysis and without dialysis: results of the PEPPER-study. PLoS One 2012: 7: e35310.

15. Humbert M, Sitbon O, Chaouat A, et al. Pulmonary arterial hypertension in France: results from a national registry. Am J Respir Crit Care Med 2006; 173: 1023-30.

16. Pecoits-Filho R, Bucharles S, Barberato SH. Diastolic heart failure in dialysis patients: mechanisms, diagnostic approach, and treatment. Semin Dial 2012; 25: 35-41.

17. Pandeya S, Lindsay RM. The relationship between cardiac output and access flow during hemodialysis. ASAIO J 1999; 45: $135-138$.

18. Vascular Access Society. Guideline 20.3: Management of high flow in AV fistula and graft. www.vascularaccesssociety.org.

19. Basile C, Lomonte C, Vernaglione L, et al. The relationship between flow of arteriovenous fistula and cardiac output in haemodialysis patients. Nephrol Dial Transplant 2008; 23 : 282-7.

20. van der Mark WA, Boer P, Cramer MJ, et al. Decreased access resistance in haemodialysis patients with upper arm arteriovenous fistulae. Nephrol Dial Transplant 2008; 23: 2105-6.

21. MacRae JM. Vascular access and cardiac disease: is there a relationship? Curr Opin Nephrol Hypertens 2006; 15: 577-82.

22. Collins AJ. Cardiovascular mortality in end-stage renal disease. Am J Med Sci 2003; 325: 163-7.

23. Smith GL, Lichtman JH, Bracken MB, et al. Renal impairment and outcomes in heart failure: systematic review and meta-analysis. J Am Coll Cardiol 2006; 47: 1987-96.

24. Adams KF Jr, Fonarow GC, Emerman CL, et al. Characteristics and outcomes of patients hospitalized for heart failure in the United States: rationale, design, and preliminary observations from the first 100,000 cases in the Acute Decompensated Heart Failure National Registry (ADHERE). Am Heart J 2005; 149: 209-16.

25. Stack AG, Bloembergen WE. A cross-sectional study of the prevalence and clinical correlates of congestive heart failure among incident US dialysis patients. Am J Kidney Dis 2001; 38: 992-1000.

26. Trespalacios FC, Taylor AJ, Agodoa LY, et al. Heart failure as a cause for hospitalization in chronic dialysis patients. Am J Kidney Dis 2003; 41: 1267-77.

27. Ahearn DJ, Maher JF. Heart failure as a complication of hemodialysis arteriovenous fistula. Ann Intern Med 1972; 77: 201-4.

28. Engelberts I, Tordoir JH, Boons ES, et al. High-output cardiac failure due to excessive shunting in a hemodialysis access fistula: an easily overlooked diagnosis. Am J Nephrol 1995; 15: 323-6.

29. Isoda S, Kajiwara H, Kondo J, Matsumoto A. Banding a hemodialysis arteriovenous fistula to decrease blood flow and resolve high output cardiac failure: report of a case. Surg Today 1994; 24: 734-6.

30. Young PR Jr, Rohr MS, Marterre WF Jr. High-output cardiac failure secondary to a brachiocephalic arteriovenous hemodialysis fistula: two cases. Am Surg 1998; 64: 239-41.

31. MacRae JM, Pandeya S, Humen DP, et al. Arteriovenous fistula-associated high-output cardiac failure: a review of mechanisms. Am J Kidney Dis 2004; 43: e17-22.

32. Stern AB, Klemmer PJ. High output heart failure secondary to arteriovenous fistula. Hemodial Int 2011; 15: 104-7.

33. Basile C, Vernaglione L, Lomonte C. Heart failure and left ventricular hypertrophy. In: Asif A, Agarwal AK, Yevzlin AS, Wu S, Beathard G eds. Interventional Nephrology, New York: McGraw Hill Medical 2012; 269-80.

34. Chemla ES, Morsy M, Anderson L, et al. Inflow reduction by distalization of anastomosis treats efficiently high-flow high-cardiac output vascular access for hemodialysis. Semin Dial 2007; 20: 68-72.

35. MacRae JM, Levin A, Belenkie I. The cardiovascular effects of arteriovenous fistulas in chronic kidney disease: a cause for concern? Semin Dial 2006; 19: 349-52.

36. London GM. Left ventricular alterations and end-stage renal disease. Nephrol Dial Transplant 2002; 17 (Suppl. 1): 29-36.

37. Wijnen E, Keuter XH, Planken NR, et al. The relation between vascular access flow and different types of vascular access with systemic hemodynamics in hemodialysis patients. Artif Organs 2005; 29: 960-4.

38. Dixon BS, Noval L, Fangman J. Hemodialysis vascular access survival: upper-arm native arteriovenous fistula. Am J Kidney Dis 2002; 39: 92-101.

39. Tordoir J, Canaud B, Haage P, et al. EBPG on vascular access. Nephrol Dial Transplant 2007; 22 (Suppl. 2): ii88-117.

40. Amerling R, Ronco C, Kuhlmann M, et al. Arteriovenous fistula toxicity. Blood Purif 2011; 31: 113-20.

41. Basile C, Lomonte C, Konner K. The arteriovenous fistula: lesser evil or God's blessing? Blood Purif 2011; 32: 253.

42. Basile C, Lomonte C. Pro: the arteriovenous fistula is a blessing of God. Nephrol Dial Transplant 2012; 27: 3752-6.

43. Vanholder R, Massy Z, Argiles A, et al. Chronic kidney di- 
sease as cause of cardiovascular morbidity and mortality. Nephrol Dial Transplant 2005; 20: 1048-56.

44. Muntner P, He J, Hamm L, et al. Renal Insufficiency and subsequent death resulting from cardiovascular disease in the United States. J Am Soc Nephrol 2002; 15: 745-53.

45. Stenvinkel P, Amann K, Ketteler M. Cardiovascular disease in chronic kidney disease. In: Feehally J, Floege J, Johnson RJ, eds. Comprehensive Clinical Nephrology, Philadelphia: Mosby Inc 2007; 839-52.

46. Shinohara K, Shoji T, Tsujimoto Y, et al. Arterial stiffness in predialysis patients with uremia. Kidney Int 2004; 65:
936-43.

47. Parfrey PS, Foley RN, Harnett JD, et al. Outcome and risk factors for left ventricular disorders in chronic uraemia. Nephrol Dial Transplant 1995; 7: 1277-85.

48. de Jager DJ, Grootendorst DC, Jager KJ, et al. Cardiovascular and noncardiovascular mortality among patients starting dialysis. JAMA 2009; 302: 1782-9.

49. Dinwiddie L. "Eligibility" is key word in Fistula First Breakthrough Initiative in determining fistula use. Nephrol News Issues 2006; 20: 39-40. 